\title{
Multiscale Brittle-Ductile Coupling and Genesis of Slow Earthquakes
}

\author{
Regenauer-Lieb*, K. \& Yuen ${ }^{+}$, D. A.
}

* Department of Earth and Geographical Sciences, The University of Western Australia, \& CSIRO, Exploration and Mining, 26, Dick Perry Ave ,WA 6102, Perth, Australia

+ Department of Geology and Geophysics \& Minnesota Supercomputer Institute; University of Minnesota, Minneapolis, MN 55455-0219, USA

We present the first attempt to explain slow earthquakes as a cascading thermal-mechanical instability. To attain this goal we investigate brittle-ductile coupled thermal-mechanical simulation on vastly different time scales. The largest scale model consists of a cross section of a randomly perturbed elasto-visco-plastic continental lithosphere on the order of $100 \times 100 \mathrm{~km}$ scale without any other initial structures. The smallest scale model investigates a km-scale subsection of the large model and has a local resolution of $40 \times 40$ $\mathrm{m}$. The model is subject to a constant extension velocity applied on either side. We assume a free top surface and with a zero tangential stress along the other boundaries. Extension is driven by velocity boundary conditions of $1 \mathrm{~cm} / \mathrm{a}$ applied on either side of the model. This is the simplest boundary condition, and makes it an ideal starting point for understanding the behaviour of a natural system with multiscale brittle-ductile coupling. Localization feedback is observed as faulting in the brittle upper crust and ductile shearing in an elastoviscoplastic lower crust. In this process brittle faulting may rupture at seismogenic rates, e.g. at $10^{2}-10^{3} \mathrm{~ms}^{-1}$ ), whereas viscous shear zones propagate at much slower rates ,up to 3 $\mathrm{X} \cdot 10^{-9} \mathrm{~ms}^{-1}$. This sharp contrast in the strain rates leads to complex short timescale interactions at the brittle-ductile transition. We exploit the multiscale capabilities from our new simulations for understanding the underlying thermo-mechanics, spanning vastly different, time -and length -scales.

\section{INTRODUCTION}

The concept of slow earthquakes [Jordan, 1991; Pfluke, 1978; Sacks, et al., 1978] has been around for nearly 30 years but there has been no serious work trying to explain the phenomenon from first principles in physics and mathematics. The recent Sumatran earthquake [Stein and Okal, 2005a; Stein and Okal, 2005b] followed by the 8.7 aftershock 
both were slow earthquakes. It is contention that many more slow earthquakes may have been undetected. Slow Earthquake instabilities, of the order of several days, have recently been reported to drop out of the numerical simulations covering millions of years of thermal-mechanical deformation [Regenauer-Lieb and Yuen, 2006]. We show here a first attempt from a solid-state point of view that can explain slow earthquakes as cascading thermal-mechanical instabilities through coupling shear heating and adiabatic heating to creep-acceleration which may explain the phenomenon. The approach is based on an extension of a classical geodynamic modelling approach, alleviating the basic shortcomings of classical, static lithosphere strength profiles, which are primarily overestimating the lithospheric strength and imply a very sharp brittle-ductile transition. The new method is based on the important positive feedbacks produced by the dissipation function, i.e. shear heating.

In order to illustrate where we might expect the maximum effect of dissipation we discuss the classical lithosphere strength profiles as proposed originally by Goetze and Evans [Goetze and Evans, 1979] and estimate where the maximum dissipation would be expected without considering feedback. The dissipation function is defined as the double dot product of the visco-plastic stress times the associated strain rate which constitutes the shear heating. "In Figure 1 we compare classical and new results shortly after application of the load showing the magnitude of the second invariant of the deviatoric stress tensor and its associated strain rate as an example for quartz/olivine rheology composite. The grey line is time-dependent and the black line is steady-state based on the constitutive relationship under application of a constant extension background strain-rate of $2.5 \times 10^{-15} \mathrm{~s}^{-1}$.

At this early stage of deformation the central part of the plate does not yet differ much from the steady state solution, however the top and bottom of the plate already show significant departure from steady-state. This is because feedback processes have already weakened the top and bottom portion of the plate. In the top $8 \mathrm{~km}$ weakening is due to the pressure sensitive yield which causes fast brittle failure. In the time-dependent solution pressure sensitive feedback has broken the upper portion sequentially from the top to the bottom within the first $1 \mathrm{k}$ years of loading. This initial response unloads the top part followed by a drop in strain rates and stress (see Figure 5). The process is rate controlled by an increase of 
the effective viscosity with depth over this pressure dependent layer (see Figure 6). The same unloading phenomenon applies to a depth greater than $50 \mathrm{~km}$, where the temperature sensitive process has weakened the bottom part of the lithosphere. Note, that in the timedependent solution weakening varies both through space and time. The phenomenon is discussed in greater details in the main body of this paper. However, the discussion is restricted to the central part of the plate for which we have sufficient numerical resolution. Failure of the central part causes the largest scale instabilities, i.e. plate size events. These largest creep events are shown to be controlled by a critical temperature in the central part of the plate which needs to be broken. The numerical simulations presented here are formulated to resolve creep instabilities for such big events. For the given background strain rate these events have a characteristic time scale $\geq 1 \mathrm{k}$ year. The calculations lack the necessary resolution to resolve smaller scale instabilities $<<1 \mathrm{k}$ years which occur in the top and bottom part of the plate. In the following the concept of the critical temperature for such plate scale instabilities is discussed on the basis of the steady state solution..

Figure 2 highlights the local critical temperature of maximum dissipation at around 900 Kelvin for olivine and 500 Kelvin for quartz assuming a local strain -rate of $2.5 \times 10^{-15} / \mathrm{s}$. At a higher strain -rate the critical temperature also goes up. The diagrams shown in Figure 1 and 2 are the classical quasi-static interpretations of strength of the lithosphere as initiated by Goetze and Evans [1979]. In this work the concept of lithospheric strength was simplified in a static picture as essentially as an elasto-plastic yield-strength envelope. The lithosphere was assumed to be elastic when loaded below the stress identified in the curve, and plastic when it reaches the curve. This obviously fixes the yield strength envelope to a particular strain rate most often $10^{-15} \mathrm{~s}^{-1}$. Later additions turned the elasto-plastic approach into a visco-elasto-plastic approach where the elastic, plastic and viscous rheological bodies are assumed to be used in series [Albert and Phillips, 2002; Chery, et al., 1991; Gerbault, et al., 2003], thus generalizing the problem for all strain-rates. 


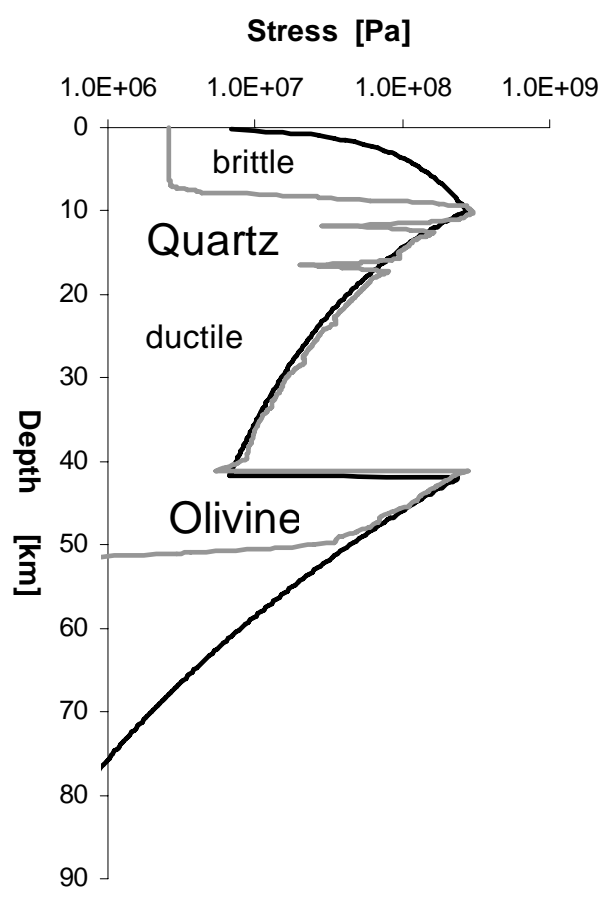

Figure 1 The classical X-mas tree (black) yield strength envelope versus a very early snapshot (130 $\mathrm{k}$ years after onset of extension) in the initial loading phase of a dynamic elasto-visco-plastic strength profile calculated in Regenauer-Lieb [2006b]. The lithosphere is made up of a simple quartz $<42 \mathrm{~km}$ depths olivine composite $>42 \mathrm{~km}$ depth. At the early stage of deformation the main difference between the two approaches is an apparent low stress near the upper and lower frictionless interfaces. Upon initial loading the upper and lower interfaces are effectively shielded elastically through the incremental elastic stretching of the areas circumscribed by the stress peaks. In subsequent deformation these stress peaks are destroyed because they are also the sites of the maximum local dissipation (Fig. 2). While the Christmas tree is a good strength measure within the classical elasto-plastic framework, a dynamic elasto-visco-plastic Christmas tree structure also requires investigation of the effective viscosity, which gives a better measure of strength through time (see Figure 5 following).

Figure 2 The associated dissipation of Figure 1 without feedback. The critical temperature is defined by maximum dissipation in the olivine/quartz power law creep regimes and is around $500 \mathrm{~K}$ for quartz rheology and $900 \mathrm{~K}$ for olivine. The classical view implies that thermalmechanical instabilities, if they exist, are to be observed at these levels.. We investigate in the following more closely the critical regime in fully coupled calculations and show that this simple concept of a critical temperature defines a gradual transition zone rather than a sharp boundary. 
This approach has, in theory, the potential of resolving a self-consistent brittle-ductile transition as a function of the imposed boundary conditions. It will, for instance, move downwards for a higher applied background strain-rate. There is, however, one significant shortcoming. The approach predicts a very sharp transition from basically elasto-plastic behaviour in the brittle crust to visco-elastic creep in the ductile regime, with no significant transition region in between. This shows the efficiency of the Arrhenius temperature dependence of the flow laws, which allows very rapid accommodation of permanent deformation by visco-elastic creep. In the extended visco-elasto-plastic transition the pressure sensitive localization mechanism thus gives abruptly away to the thermally accommodated creep. This is at odds with inferences from both laboratory experiments [Kohlstedt, et al., 1995] and the field [Mancktelow, 2006]. Transitional brittle-ductile behaviour develops naturally, when one considers the positive feedback from the dissipation function .

The first fully coupled thermal-mechanical approach in geology was pioneered by Chery et al. [1991], however, at that early stage the required spatial resolution was still sorely lacking and thermal expansion was not considered, hence the effects of feedback reported by this work was overlooked. The first model with potential relevance to slow earthquakes was published [Regenauer-Lieb and Yuen, 2006]. In that paper we analysed a single quartz crust in extension and found self-consistent development of three distinctly different rheological layers: a fully brittle layer at the surface with fast time scale instabilities, a bottom fully ductile layer with slow mylonitic shear zones and an intermediate semibrittle/ductile layer of several km thickness, where processes communicate. For the case of quartz rheology this resulted in a single critical temperature with maximum dissipation governing the creep/brittle ductile layer instability system. Here, we extend the analysis to the lithosphere and perform a more detailed analysis of the brittle-ductile coupling. 


\section{Modelling Framework}

We use a fully coupled thermo-mechanical framework for the generation of shear zones in geology and planetary sciences [Regenauer-Lieb and Yuen, 2003]. The key element of the theory is an incorporation of the energy equation as a master of the two driving forces for instability, which are the pressure pulses $\Delta \mathrm{P}$ and temperature pulses $\Delta \mathrm{T}$ acting through the filter of continuity and the quasi-static momentum equation as slaves. Both temperature and pressure pulses are positive feedback variables giving rise to the instabilities. The only negative feedback constraints preventing catastrophic failure are given through conduction, dissipating the thermal pulse, and imposed boundary conditions stabilizing the pressure through the quasi-static momentum and the continuity equations. Figure 3 shows the general framework of the brittle/ductile feedback loop where the equation of state is tied to the continuity and the rheology acts as the most important feedback filter. The temporal evolution of the process is thus controlled inherently by the thermodynamic evolution of the energy equation. A more detailed description of the feedback loops, the equations employed and a comparison to the classical engineering style approach, which only considers moment and continuity equation, can be found elsewhere [Regenauer-Lieb and Yuen, 2004].

We want to point out at this stage that the classical theory neglects the energy evolution, ,which is at the heart of any non-linear timescale, such as occurring in earthquakes. Attempts to incorporate time scale through empirical strain-, or strain rate weakening laws or rate and state variable friction [Dieterich, 1979a; 1979b] are not self-consistent within the framework of the energy equation. The empirical laws may be extended to a continuum framework [Ord, et al., 2004], however, careful examination of the energy equation needs to be performed, such as other sources of energy due to grain-boundary processes [Bercovici and Ricard, 2003]. The main drawback is that an empirical plasticity framework does not prevent violation of the energy equation, which, incidentally may occur. The original CAM-Clay model [Collins and Hilder, 2002] is a typical example which has been shown to violate the second law of thermodynamics. Although the energy equation is not 
explicitly needed in such isothermal models, an amendment in a thermo-mechanical framework is mandatory. The incorporation of (isothermal) thermodynamics into mechanics pioneered in Zieglers book [Ziegler, 1983] has recently revolutionized plasticity theory and the modelling shallow deformation in soil and sands [Collins, 2005]. This example shows the key role of the energy equation for geological applications where we are certainly not dealing with isothermal conditions.

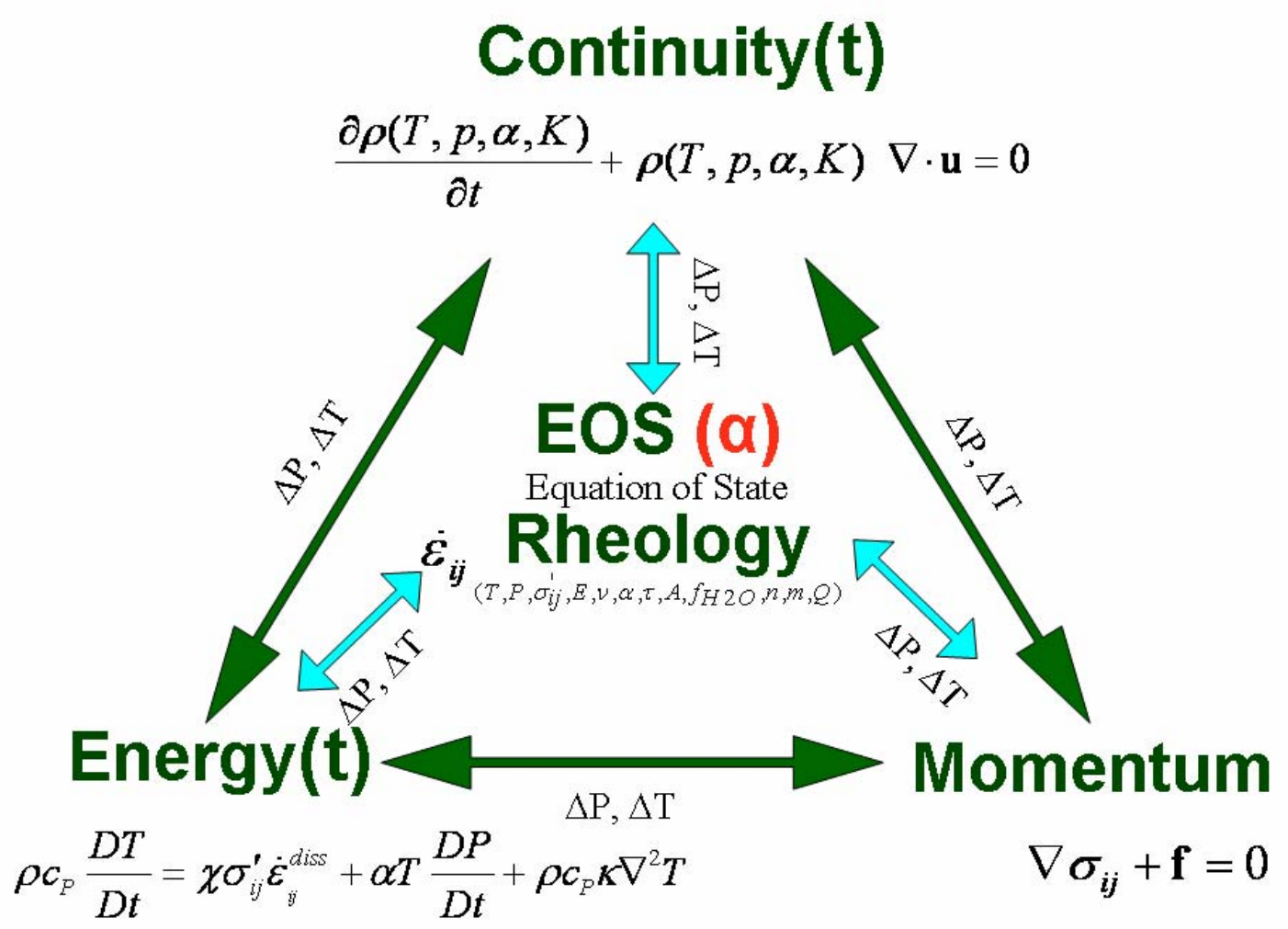

Figure 3 Small changes in pressure and temperature can have a large effect on the deformation of rocks. Nonlinear feedback can cause pattern formation out of an initially unpatterned state. Here, two important mechanical feedback processes are described in the energy equation. Small perturbations in temperature and pressure are caused by shear heating (first term on the right side of the energy equation), thus changing the entropy through dissipation and thermal expansion (second term) being an isentropic process. Both dissipative and non-dissipative processes can cause formation of shear zones. The time-dependence in both the energy and continuity equations is indicated by the symbol $\mathrm{t}$. 


\section{Equations solved}

\section{Continuity Equation}

We express the continuity equation here in differential form where the scalar (dot) product of the velocity vector $\mathbf{u}$ with the nabla vectorial differential operator $\nabla$ i.e. the divergence of the velocity field is exactly equal to the negative rate of the fractional density change.

\section{Equation 1}

$$
-\frac{1}{\rho} \frac{D \rho_{(T, P, \alpha \ldots)}}{D t}=\nabla \cdot \mathbf{u}
$$

here $D / D t$ is the substantive Lagrangian (also called material, Lagrangian, convective) derivative. The density $\rho$ of the solid Earth materials is itself is a function of the thermodynamic state variables, the most important are temperature $T$, pressure $P$ and strain caused by thermal expansion $\alpha$. The substantive derivative is the rate of change of some material property (here the density) defined by:

$$
\frac{D()}{D t}=\frac{\partial()}{\partial t}+\mathbf{u} \cdot \nabla()
$$

where $\mathbf{u}$ is the local material velocity vector of the volume under consideration and the empty bracket is a placeholder for the particular material property. This can be rearranged considering the dependence of the density on the thermodynamic state variables as

$$
\frac{\partial \rho_{(T, P, \alpha \ldots)}}{\partial t}+\left(\nabla \rho_{(T, P, \alpha \ldots)} \cdot \mathbf{u}\right)=0
$$

Equation (1) incorporates time as a derivative, which is implicitly derived from the evolution of isentropic work to be discussed in conjunction with the energy equation. This subtlety of continuity equation enhances thermal-mechanical coupling which is of course not present in the incompressible approaches, where time does not enter into the continuity equation. We emphasize that volumetric deformation cannot be neglected, especially in 
solid-state phase transitions, such as the basalt to eclogite transition for geodynamic conditions.

\section{Momentum Equation}

In its general form the material derivative also enters the Momentum equation there describing the accelerating components experienced by the Lagrangian (i.e.convected) material volume. In geodynamic deformation it is a common practice to drop the inertial forces, which contain the time-dependence. Whether this approach still holds for slow earthquakes is questionable. However, we shall adopt here as a first approach the common practice of neglecting inertial terms. With this simplification the momentum equation therefore can be written independent of time as the equilibrium condition of the surface tractions (divergence of the Cauchy stress tensor $\sigma_{i j}$ ) applied over the surface of a volume element and the body forces $\mathbf{f}$ applied to the volume element.

\section{Equation 2}

$\nabla \cdot \sigma_{i j}\left(T, P, \sigma_{i j}^{\prime}, E, v, \alpha, \tau, A, f_{H 2 O}, n, m, Q\right)+\mathbf{f}=0$

The Cauchy stress tensor is in itself a function of the rheology (equation 3), depending in our calculations on the variables temperature $T$, pressure $P$, deviatoric stress $\sigma_{i j}^{\prime}$, Young's modulus $E$, the Poisson's ratio $v$, the volumetric coefficient of thermal expansion $\alpha$, the plastic yield stress $\tau$, and the creep law parameters, such as the activation enthalpy and preexponential factor (equation 3). The most important quantities in terms the creep law are the water fugacity $f_{H 2 O}$ and the activation enthalpy $Q$ while $\mathrm{A}, \mathrm{m}$ and $\mathrm{n}$ are the parameters for the phenomenological fit to the creep behavior. 


\section{Rheology}

For ductile and brittle behavior, we use a composite rheology by assuming a modified von Mises plasticity, with a pressure dependent yield stress (linear Drucker-Prager) combined with the elasto-visco-plastic coaxial flow rule. This gives the following:

\section{Equation 3}

$\dot{\varepsilon}_{i j}=\left(\frac{1+v}{E} \frac{D \tilde{\sigma}_{i j}^{\prime}}{D t}+\frac{v}{E} \frac{D P}{D t}+\alpha \frac{D T}{D t} \delta_{i j}\right)_{\text {elastic }}+\left(\dot{\varepsilon}^{p l} \frac{\sigma_{i j}^{\prime}}{2 \tau}\right)_{\text {plastic }}+\left(A f_{H_{2} O}^{m} \sigma_{i j}^{\prime} J_{2}^{n-1} \exp \left(-\frac{Q}{R T}\right)\right)_{\text {creep }}$

Recall that $E$ is Young's modulus and $v$ is Poisson ratio. $\dot{\widetilde{\sigma}}_{i j}$ is the objective co-rotational stress rate (refer to the Appendix on the numerical technique) and $\delta_{i j}$ is the Kronecker delta. $J_{2}$ is defined as the second invariant of the deviatoric stress tensor:

$J_{2} \equiv \sqrt{\frac{3}{2} \sigma_{i j}^{\prime} \sigma_{i j}^{\prime}}$

The plastic yield potential $\Phi$ defines the elasto-plastic limit at low pressure. It is known as a linear Drucker-Prager Yield Criterion, whereby the coefficient for $P$ is assumed to be one and the tensile strength is assumed zero.

$\Phi=J_{2}-P=0$

Inside the potential function the material response is entirely elastic (plus some minor viscous flow) and when the stress reaches values defined by the yield potential plastic strain rates are allowed. These are also based on the second invariant $J_{2}$. The Drucker-Prager law therefore is coaxial meaning that the plastic stress and strain-rates have the same principle axis, and the magnitude of the yield stress is scaled by the magnitude of the first invariant $\mathrm{P}$.

\section{Energy Equation}

The substantive derivative makes a prominent reappearance in the energy equation 4 as both temperature $T$ and pressure $P$ convected derivatives. A Lagrangian mesh is therefore chosen for our numerical solution. 


\section{Equation 4}

$\rho c_{P} \frac{D T}{D t}=\chi \sigma_{i j}^{\prime} \dot{\varepsilon}_{i j}^{d i s s}+\alpha T \frac{D P}{D t}+\rho c_{P} \kappa \nabla^{2} T$

The total change of heat balances in three important terms. The first two terms on the right hand side of equation 4 describe the temperature change due to dissipative and stored mechanical work, respectively. The first term on the right is also known as the shear heating term where $\chi$ is the efficiency of converting mechanical work into heat at large deformation $\chi$ is of the order of 0.9 for most materials[Chrysochoos and Dupre, 1992]. Shear heating only describes the temperature changes owing to dissipation where $\dot{\varepsilon}_{i j}^{\text {diss }}$ is the plastic and viscous strain rates. The second (isentropic) term on the right describes the reversible temperature change due to elastic volumetric changes it thus contains the effect of temperature changes due to elastic compressibility. The third term gives the familiar contribution of heat conduction being a function of diffusivity $\kappa$ and $\nabla^{2}$ is the Laplacian scalar differential operator. The specific heat of the Lagrangian convected volume element is $c_{p}$.

\section{The Geodynamical Model}

We investigate a very simple but basically fundamental model where a $100 \mathrm{~km}$ long segment of continental lithosphere is subject to far field extension of $0.5 \mathrm{~cm} / \mathrm{a}$ applied on either side. The continental lithosphere consists of a $42 \mathrm{~km}$ thick quartzitic crust underlain by peridotitic mantle material with a $38 \mathrm{~km}$ thickness. At the bottom and along the sides of the lithosphere free slip, i.e. zero tangential stress boundary conditions are assumed, while the displacements are constrained by zero vertical displacement on the bottom and on the side only periodic vertical displacements are allowed, i.e. the vertical displacements on the right are constrained to be exactly equal to the vertical displacement on the left-hand side of the model. The top boundary is an open boundary.

The thermal boundary conditions are also very basic. At the bottom of the lithosphere segment, a fixed base mantle heat flow of $30 \mathrm{~mW} / \mathrm{m}^{2}$ is assumed while within the crust, there is curvature following the steady state for continents. The initial thermal profile is allowed to be in the steady state limit. Superposed on this classical pattern are small random thermal perturbations, allowing a white noise seed for nucleating the shear zones. 
Results: 1. Geological Time Scale (O ( 10 Ma) : We are here describing only modelling results for the case of extension. The modelling results for compression underline the identification of (higher) critical temperature for transition from brittle to ductile processes, but they display different complex patterns not discussed here. Continental breakup has been followed to formation of an ocean (time scale, millions of years. For reasons of clarity we do not focus on geodynamic consequences of a switch from a crustal quartz strength to a mantle olivine strength control [Rosenbaum, et al., 2006] observed for lower surface heat flow. In Figure 4 we first discuss a simple calculation of quartz crust with $60 \mathrm{~mW} / \mathrm{m}^{2}$ surface heat flow and a frictionless base instead of a mantle layer [Regenauer-Lieb and Yuen, 2006]. We use this example to investigate in more details the possible thermalmechanical mechanism underlying the generation of short-time scale instabilities.

For classical extension models, that do not consider brittle-ductile energy feedbacks, steeply dipping normal faults are conventionally obtained [Wijns, et al., 2005]. Listric, normal faults, as observed in geology, are very difficult to achieve. Our fully coupled simulation overcomes this problem [Regenauer-Lieb, et al., 2006b]. Localization feedbacks in and between the brittle and ductile layers can turn the direction of faults around. Listric faults are caused by efficient weakening on brittle-ductile detachment layers. These subhorizontal layers develop through shear heating forming brittle and ductile flow localization. Two detachments are defined by a bottom mylonitic zone formed by ductile localization and a top detachment zone due to brittle localization. Upon inspecting the more detailed structure of the detachment layer, we encounter a surprising complexity all located within the brittle-ductile transition zone, which upon initial loading forms an elastic core. The core is ruptured from two sides (Figure 4). From the top the propagation of a brittle fault is driven by the combination of loading and brittle localization feedback effects. Fault propagation is hampered, as it reaches the top of the elastic core, where brittle and ductile processes interact, because a viscous response blunts the brittle fault tip and eliminates the stress singularity. Further arrest of the brittle fault is driven by ductile thermal-mechanical feedback effects, which act to decrease the differential stress at the tip of the fault by lowering the effective viscosity. Simultaneously, ductile shear zones propagate upwards towards the elastic core. However, these shear zones die out because of increasing crustal 
strength and because they reach the area in which brittle localization feedback dominates. Their energy is translated into a local, positive amplitude disturbance of the elastic stress field. This extra stress, loaded to the core from below, eventually starts to interact with similar features loaded from above.
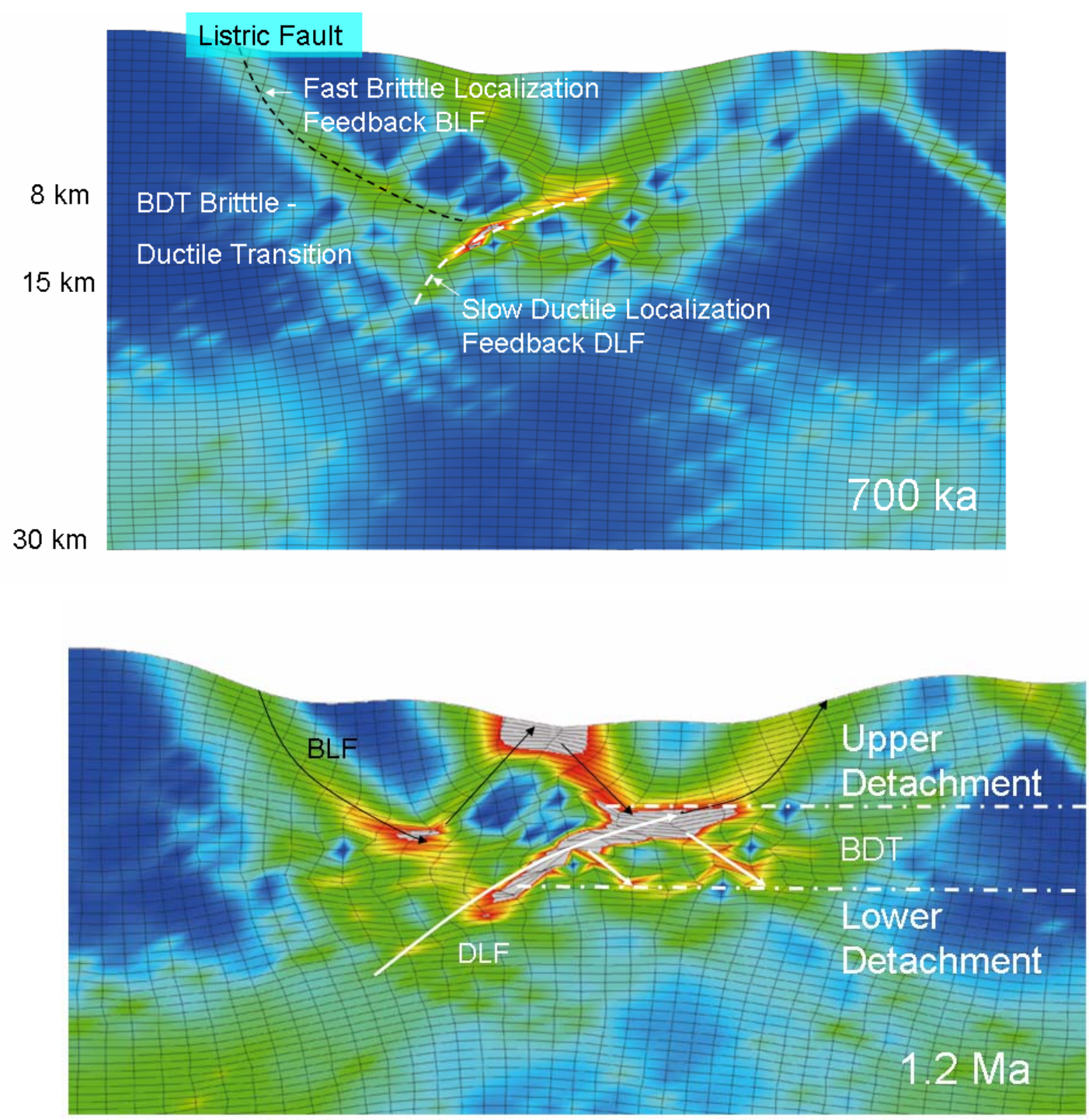

Figure 4 Brittle (P-dependent) and ductile (T-dependent) feedback for a small piece of continental crust in extension illustrating the brittle-ductile feedback. Two subsequent time slices are shown ,700 k and $1.2 \mathrm{Ma}$ after the elastic loading phase. The thermal conditions are $60 \mathrm{~mW} / \mathrm{m}^{2}$ surface heat flow and the total extension velocity for this particular model is $2 \mathrm{~cm} / \mathrm{yr}$. Only the crust is considered, for simplicity. The two detachments are delimiting an area to either side of the stress and maximum dissipation peaks in Figure 1 and 2 , thus decoupling an "elastic core" of the crust , i.e. an elastic stress bearing portion of the crust. This maximum elastic energy and maximum dissipation area plays a significant role for slow earthquake instabilities. 
We now come back to the discussion of the time evolution of the $70 \mathrm{~mW} / \mathrm{m}^{2}$ lithospheric model case presented in Figure 1. In a dynamical framework the strength envelope of Figure 1 involves not only a discussion of stress but also the strain-rates. We propose that a suitable measure of strength is the effective viscosity. The stress/strain rate - depth curve for the dynamic curve in Figure 1 is shown in Figure 5 and the resultant viscosity profile is shown in Figure 6.

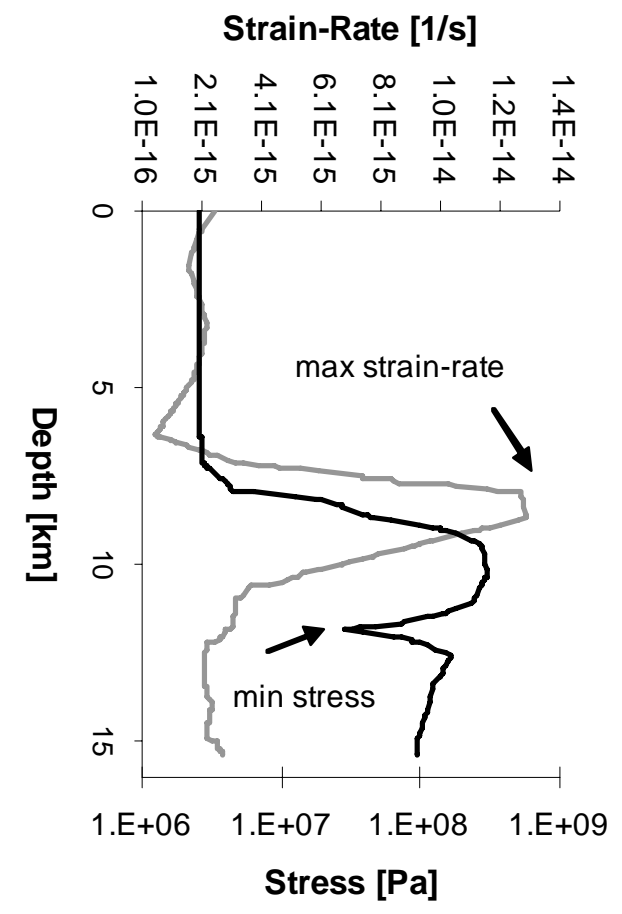

Figure $5 \log$ (Stress) (black) and $\log ($ StrainRate) (grey) versus depth curve showing data from the dynamic strength-evolution model shown in Figure 1, focussing on the brittle-ductile transition during the initial elastic loading phase after $130 \mathrm{kyrs}$ extension with a background strain-rate of $2.210^{-15} \mathrm{~s}^{-1}$. A $2.5 \mathrm{~km}$ phase shift between the maximum strain-rate and the maximum stress peak is visible. The upper detachment develops at the maximum in strain-rate at around $8.5 \mathrm{~km}$ depth while the lower detachment develops at the minimum in the local stress at around $12 \mathrm{~km}$ depth. 


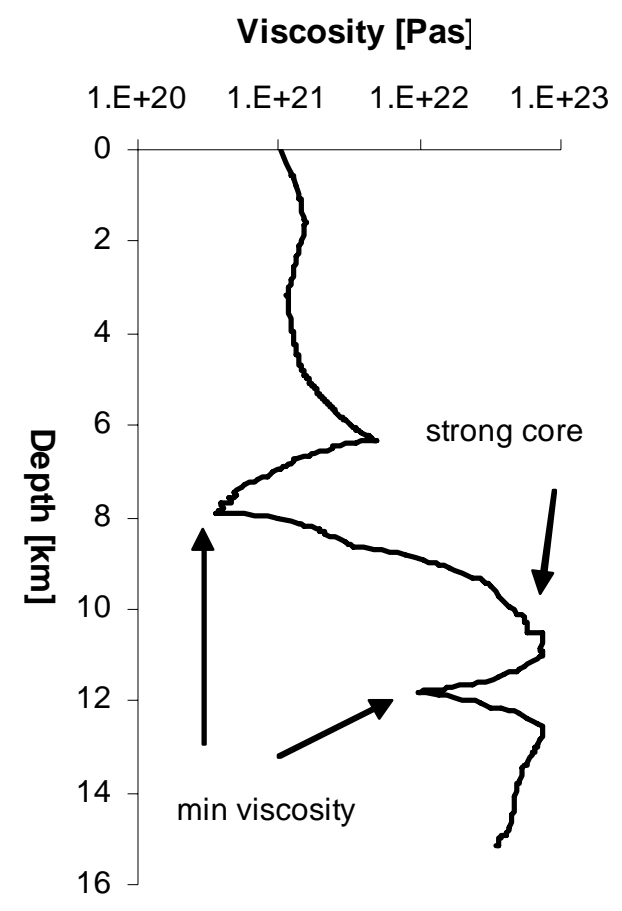

Figure 6 Log (viscosity) versus depth of the model shown in Figure 5. The effective (secant) viscosity is defined by dividing the $2^{\text {nd }}$ invariant of the stress over its associated strain rate. The two detachments around the "elastic" stress bearing core are clearly visible as local viscosity lows. At the early stage of deformation there is still significant strength in the ductile part of the crust.

At the onset of deformation (e.g. 130 kyrs) the energy feedback affects mainly the maximum dissipation depth level shown in Figure 2 thus chiefly depends on depth/temperature. A 1-D depth profile might therefore give representative results for the entire model. The more evolved strength evolution curves depend not only on depth but also significantly on the horizontal dimension. For instance, near the surface in the vicinity of fully developed listric shear zones shown in figure 4, the stress is significantly higher than in the center of the graben structure, away from this shear zone. For ease of comparison to Figure 5 and 6 we monitor the 1-D depth-profile in a vertical cross section through the stable middle of the graben structure, thus resolving mainly vertical changes in strength evolution for the evolved stage after 6.4 Ma of extension in Figure 7 and 8. 


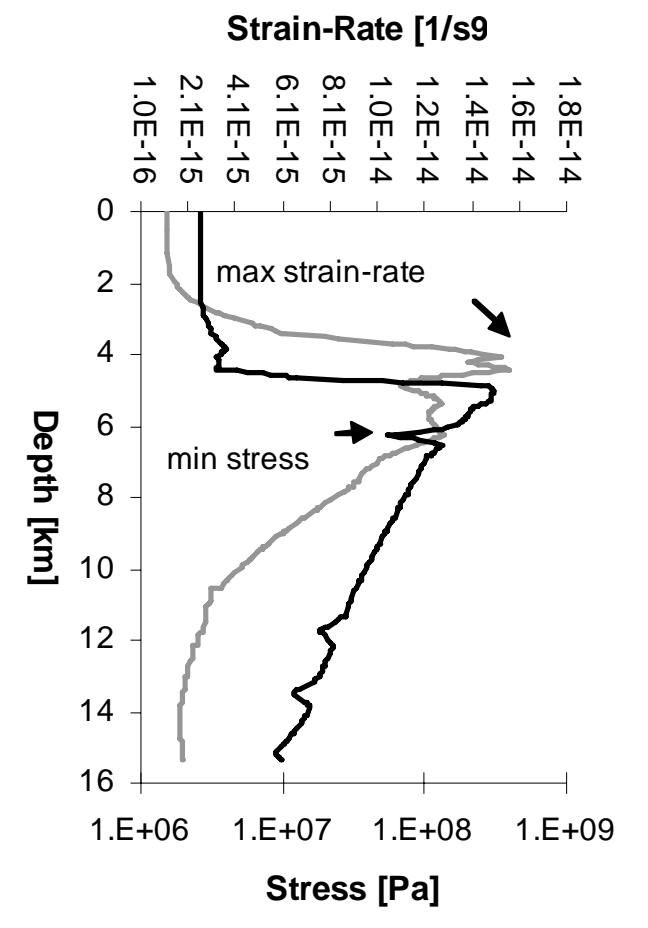

Figure 7 Log (Stress) (black) and $\log ($ StrainRate) (grey) versus depth curve showing data from the dynamic strength-evolution model shown in Figure 5 after 6.4 Myrs of extension. The phase shift from maximum in strain-rate to maximum in stress still persists. However, the shift is reduced to $1 \mathrm{~km}$. Consequently the two detachments near the maximum in strain-rate and the other local maximum at the local minimum in stress are approaching each another. 


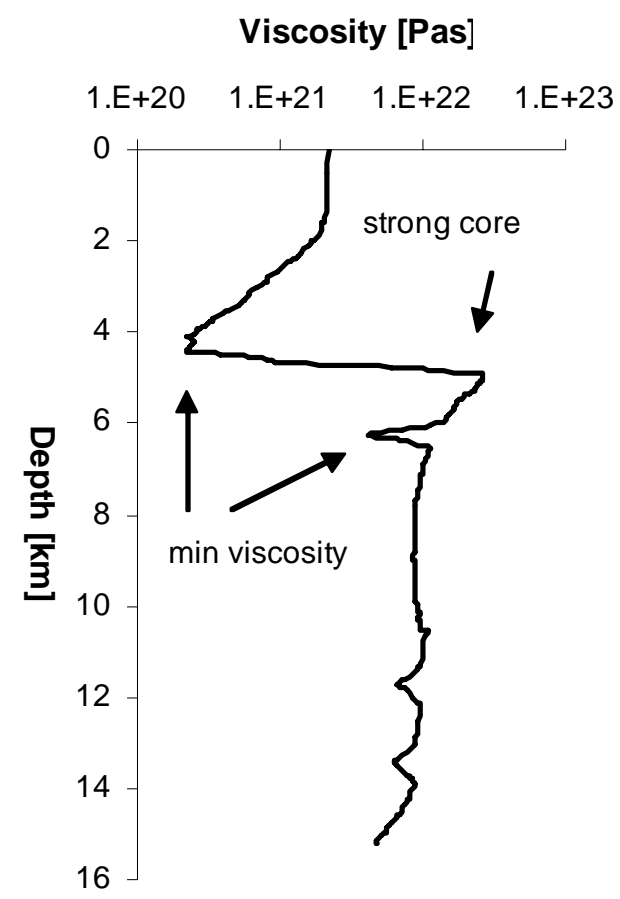

Figure 8 Log (viscosity) versus depth of the model shown in Figure 7. There is a pronounced viscosity minimum near the upper detachment. The lower crust contributes significantly to the strength of the crust, however, not as significant as the strong core, which is very small and located at only $5 \mathrm{~km}$ depth.

Figure 7 and 8 show a consistent evolution of features visible in Figure 5 and 6 . The main feature is a vertical upwards movement of the detachment accompanied by a narrowing of the upper and lower detachments. The two shear zones sometimes coalesce through individual oblique shear zones, to the effect that the core is disrupted during these creep burst events. In this way, the upper mid-crustal detachment zone, develops into two low viscosity zone, a defined by two detachments separated by an elastic core. We argue that the rupture behaviour of this elastic core holds the key to crustal earthquakes. In order to zoom into the dynamics associated with the critical behavior, we investigate a small sub-model representing a thin depth slice of the continental crust with a spatial resolution of $40 \mathrm{~m}$.

Results 2. Geodetic Time Scale ( O( 1 Ma) : Crustal deformation (time scale years) is compared to high resolution geology. For this first analysis a depth slice is taken with isothermal boundary conditions using stress and displacement boundary conditions from the large scale model. The thermal boundary conditions are different to the large scale model. This model has random thermal perturbation of $70 \mathrm{~K}$ corresponding to the approximate amplitude of a natural thermal gradient of a $3 \mathrm{~km}$ vertical slice of the crust. This 
perturbations are inserted as random white noise on $4 \%$ of the nodes with a maximum positive amplitude of $70 \mathrm{~K}$. After 2 Million years the thermal diffusion has reduced the perturbation to an amplitude of only about $10 \mathrm{~K}$. The wavelength of the thermal perturbations after deformation is not only controlled by conduction but also significantly affected by the thermal-mechanical feedback. We use a homogeneous setup with a central layer of aplitic material in the middle of the crustal granitic slice. The background temperature is varied in individual model runs in $10 \mathrm{~K}$ increments from $430 \mathrm{~K}$ to $560 \mathrm{~K}$. In this first test of the mechanical response the sub-model is not advected within a large-scale crustal model, i.e. not fully coupled to the large scale simulation. It therefore lacks the dynamics of the brittle-ductile transition discussed above.

We are interested for this paper only in the material behavior around the critical temperature, i.e. the material response that leads to the rupture of the elastic core. At this early stage we also wish to isolate the meso-scale material response from the large scale hierarchical driver. An exactly analogous analysis is done under convergent boundary conditions and reported elsewhere [Regenauer-Lieb, et al., 2006a]. Future follow up studies will also look into full coupling of geodetic and geodynamic time- and lengthscales. First, we will search for exemplary regions of interest.

The meso-scale results clearly show that in addition to the afore described $\mathrm{P}$ - and $\mathrm{T}$ dependent brittle/ductile fault/shear zones above and below the brittle ductile transition we record near a critical temperature an additional phenomenon that deviates significantly from the pure shear boundary conditions. Localized centers with high vorticity appear most prominently in the feldspar layer (Figure 9 and 10). High elastic energy inside the vortices are the sources of short-time scale instabilities. Figure 9 shows that these centers are controlled by the thermal expansion feedback and relate to local temperature maxima. Figure 10 shows that the local vortices store a significant amount of elastic energy. 

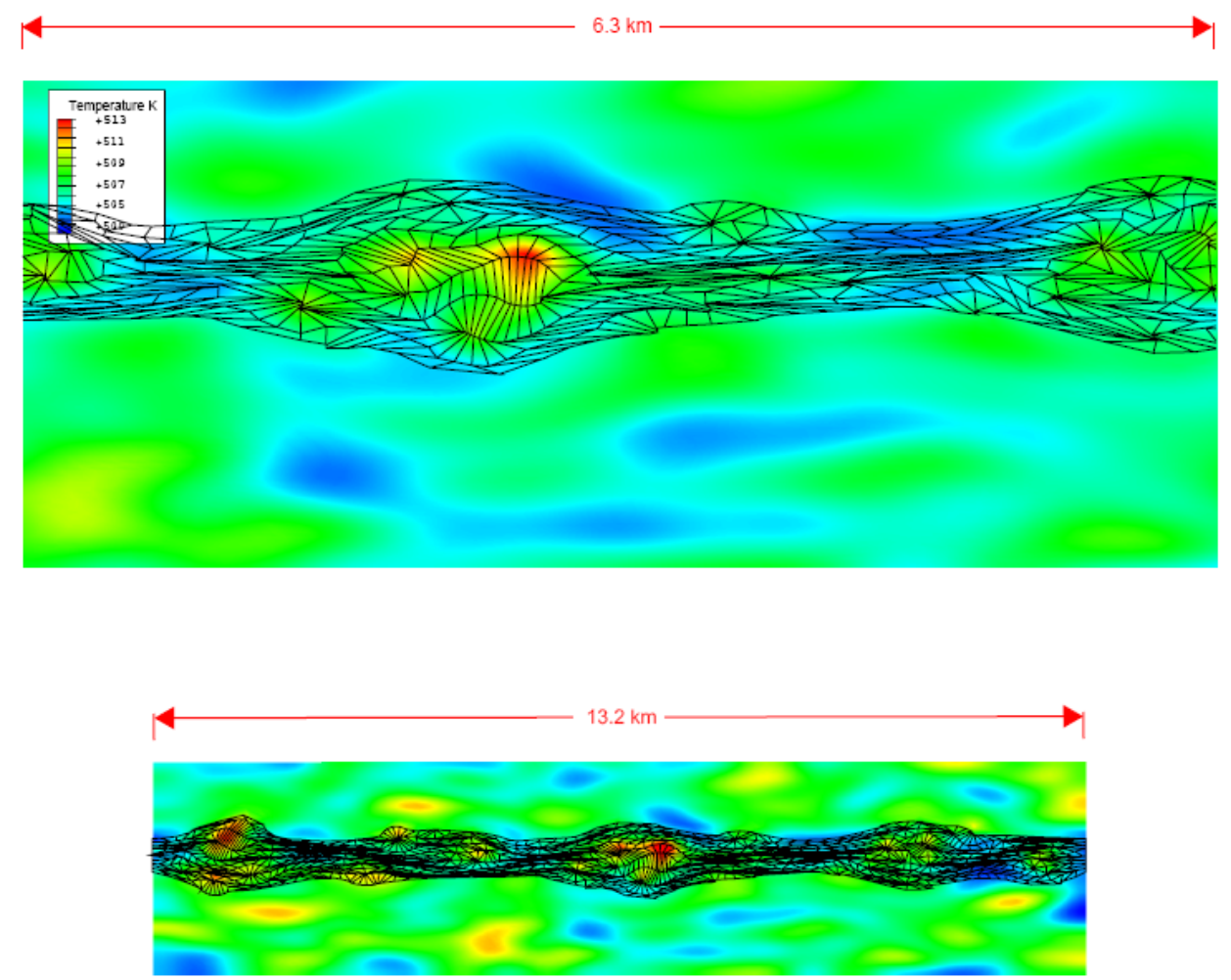

Figure 9 Temperature distribution after 2 Million years of extension. The original rectangular grid over the feldspar layer shows significant distortion and rotation around temperature maxima.
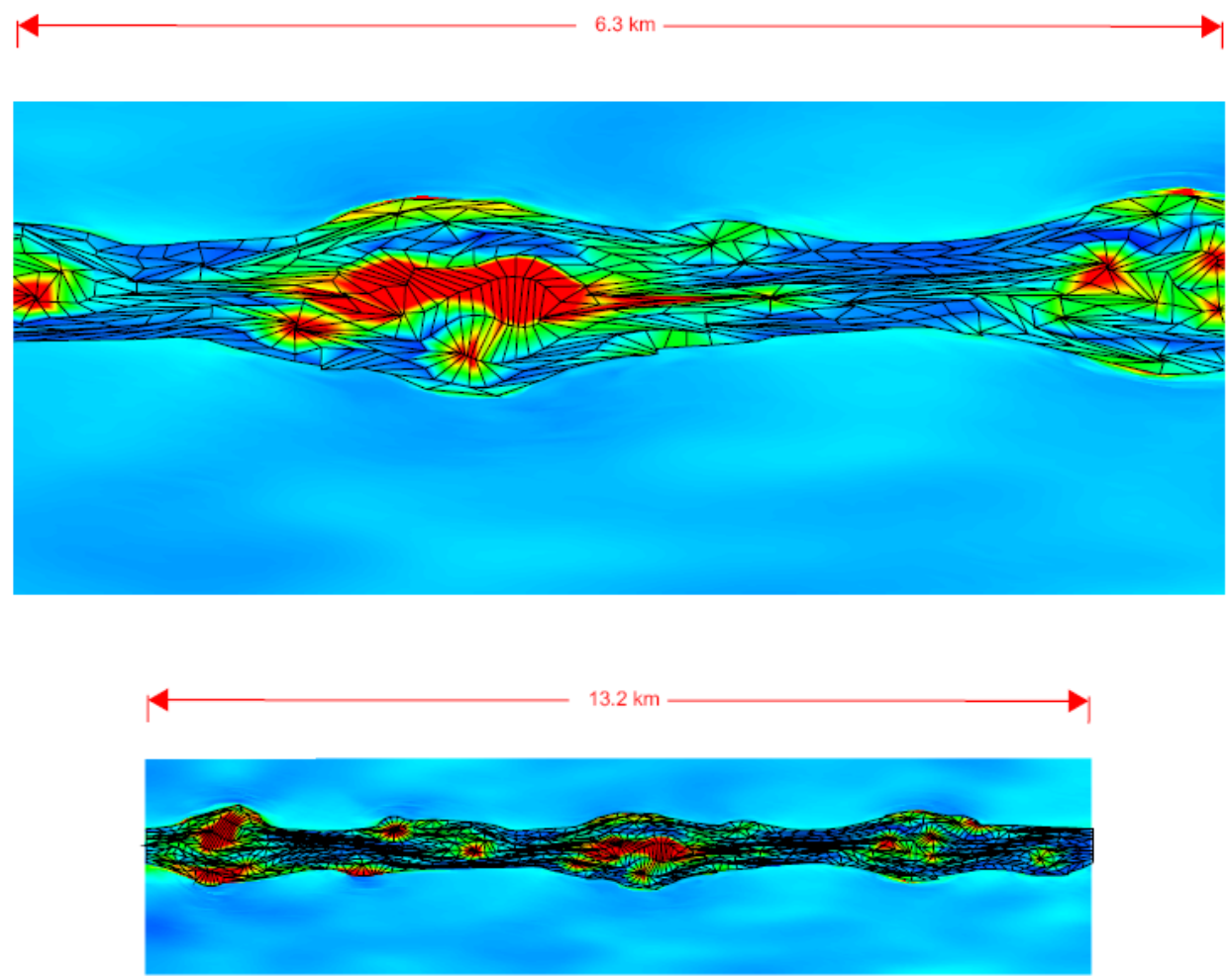

Figure 10 Elastic energy distribution ( $\mathrm{red}=1.010^{6} \mathrm{~J} / \mathrm{m}^{3}$ ) corresponding to Figure 9 . The central portion of the high temperature vortices stores a significant amount of elastic energy. 


\section{Towards Earthquake Timescales O (a) :}

We are now putting the above calculations into perspective for the type of time-scale relevant for earthquakes without presenting additional results. Obviously, a conclusive analysis can only be done if geodynamic and geodetic length and time scales are fully coupled in a multi-scale framework. This analysis would require robust automated adaptive meshing techniques, available for the entire critical shear zone, where the discretization needs to be smaller than the thermal diffusional length-scale associated with the thermal disturbances within the recurrence interval of major earthquakes, say 1000 years. This time interval amounts to the length-scale of a soccer field, i.e. around $100 \mathrm{~m}$. Such an analysis is feasible. However, we would additionally wish to consider inertial terms to explore the richer dynamics at smaller time-and length scale. Our approach is clearly at a very early stage. However, we would argue that the results are nonetheless pointing into a promising avenue of research and highlight possible application to the genesis of slow earthquakes, which we discuss below.

Extraction of the free-oscillation amplitude excited by the recent Sumatran earthquake by Stein and Okal [2005a] has shown a linearly growing trend in the semi-log plot between amplitude and frequency. This trend is reminiscent of the Rayleigh-Jeans portion of the Planck curve in radiation physics, which was posed as the so-called ultra-violet catastrophe. This signature shows that some other physics must intervene to neutralize this singular tendency. This physics obviously controls the largest size of the slow slip event which we argue to be in turn controlled by the energy stored in the elastic core and the thermalmechanical rupture behaviour of the core. We postulate here that the centers of vorticity recorded in the meso-scale simulation could be a possible candidate for the analogy of a "Plancks Quant" for earthquakes. We call attention to the large-scale simulation shown in Figure 4 which also shows these vortices in between the upper and lower detachment. It is hence a robust feature for a crust in extension for both geodynamical and geodetic timescale simulations. 


\section{Summary and Future Perspectives}

Our study presents thermal-mechanical shear interaction within the framework of a twodimensional time-dependent model wherein a realistic visco-elastic-plastic rheology is employed, and the governing equations include the momentum equation without inertia, the rheological and energy equation. Earlier work on one-dimensional model with the Peierls stress built into the rheology [Kameyama, et al., 1999] has already shed some light on these short-timescale instabilities. Other work pioneering the two dimensional dynamics caused by rheological layering have also been suggested[Lyakhovsky, et al., 2001]. The models presented here make the case more convincing. In the energy equation we have retained all mechanical heating terms and heating terms involving volumetric expansion. In our simulations we encounter two basically different bifurcation phenomena at the brittleductile transition-zone, which can be attributed to two different families of eigenmodes of the system. One in which the shear zone nucleates on thermal perturbations in the ductile field, and the second which is fully associated with elasto-plastic (brittle, pressuredependent) displacements associated with thermal stresses. A quartz slab has all two modes operating simultaneously in three different depth levels. The bottom of the crust is controlled by the elasto-visco-plastic mode while the top is controlled by the elasto-plastic mode. The exchange of the two modes appears to communicate on a sub-horizontal layer within the brittle-ductile transition zone. We show that the isentropic work term, in the energy equation, encapsulating the effect of thermal-elastic expansion, is the key to fast time scales of deformation. Geodetic meso-scale simulations show that thermal expansion is particularly important in a critical temperature domain at the brittle-ductile transition where the two different eigenmodes interact. Near the critical temperature thermal expansion forms local centers of vorticity having a length scale of the order of several hundred of meters. In our calculations these centers are controlled by both heat conduction feeding back through shear heating and thermal expansion through individual creep burst events. We demonstrate here the underlying mechanism for fast timescale instabilities to be the thermal expansion work from coupling to elasticity. Therefore, we have shown that with a properly formulated thermal-mechanical model, we can generate timescales now very close to those of earthquakes and of the same order as slow earthquakes. These results 
together with future data acquisition from GPS data would help to link the two regimes of timescales and enable us to develop a uniformly valid rheological law for the lithosphere.

Acknowledgements

We thank discussions with Bruce Hobbs and Charley Kameyama. This research has been supported by NSF's ITR and Math-Geo programs and the Western Australian Premiers Fellowship program, the University of Western Australia and the CSIRO Exploration and Mining Division.

\section{References:}

Albert, R. A., and R. J. Phillips (2002), Time-dependent effects in elastoviscoplastic models of loaded lithosphere, Geophysical Journal International, 151, 612-621.

Bercovici, D., and Y. Ricard (2003), Energetics of a two-phase model of lithospheric damage, shear localization and plate-boundary formation, Geophysical Journal International, 152, 581-596.

Chery, J., et al. (1991), Thermomechanical Evolution of a thinned continental lithosphere under compression: Implication for the Pyrenees, Journal of Geophysical Research, $96,4385-4412$.

Chrysochoos, A., and J. C. Dupre (1992), An infrared set-up for continuum thermomechanics, Journal of Societe Francaise des Thermiciens, 27, 129.

Collins, I. F. (2005), Elastic/plastic models for soils and sands, International Journal of Mechanical Sciences, 47, 493-508.

Collins, I. F., and T. Hilder (2002), A theoretical framework for constructing elastic/plastic constitutive models of triaxial tests, International Journal for Numerical and Analytical Methods in Geomechanics, 26, 1313-1347.

Dieterich, J. H. (1979a), Modeling of Rock Friction .1. Experimental Results and Constitutive Equations, Journal of Geophysical Research, 84, 2161-2168.

Dieterich, J. H. (1979b), Modeling of Rock Friction .2. Simulation of Pre-Seismic Slip, Journal of Geophysical Research, 84, 2169-2175.

Gerbault, M., et al. (2003), Numerical models of lithospheric deformation forming the Southern Alps of New Zealand, Journal of Geophysical Research B: Solid Earth, 108.

Goetze, C., and B. Evans (1979), Stress and temperature in the bending lithosphere as constrained by experimental rock mechanics, Geophysical Journal of the Royal Astronomical Society, 59, 463-478.

Jordan, T. (1991), Low-frequency characteristics of slow earthquakes and their implications for near-field monitoring of precursory phenomena, Seismological Research Letters, 62, 40.

Kameyama, C., et al. (1999), Thermal-mechanical effects of low temperature plasticity (the Peierls mechanism) on the deformation of a viscoelastic shear zone, Earth and Planetary Science Letters, 168, 159-162.

Kohlstedt, D. L., et al. (1995), Strength of the lithosphere: Constraints imposed by laboratory measurements, Journal of Geophysical Research, 100, 17587-17602.

Lyakhovsky, V., et al. (2001), Earthquake cycle, fault zones, and seismicity patterns in a rheologically layered lithosphere, Journal of Geophysical Research-Solid Earth, 106, 4103-4120.

Mancktelow, N. S. (2006), How ductile are ductile shear zones?, Geology, 34, 345-348. 
Ord, A., et al. (2004), A smeared seismicity constitutive model, Earth, Planets and Space, $56,1121$.

Pfluke, J. (1978), Slow earthquakes and very slow earthquakes, edited by M. P. U. S. Geol. Surv., Calif., United States (USA), pp. 447-468, Open-File Report - U. S. Geological Survey.

Regenauer-Lieb, K., et al. (2006a), Depth-Frequency distribution and a new brittle-ductile mechanism for crustal earthquakes, Nature, in prep.

Regenauer-Lieb, K., et al. (2006b), The effect of energy feedbacks on continental strength, Nature, 442, 67-70.

Regenauer-Lieb, K., and D. Yuen (2006), Quartz Rheology and short time-scale crustal instabilities, Pure and Applied Geophysics, 163.

Regenauer-Lieb, K., and D. A. Yuen (2003), Modeling Shear Zones in Geological and Planetary Sciences: Solid- and Fluid- Thermal- Mechanical Approaches, Earth Science Reviews, 63, 295-349.

Regenauer-Lieb, K., and D. A. Yuen (2004), Positive feedback of interacting ductile faults from coupling of equation of state, rheology and thermal-mechanics, Physics of Earth and Planetary Interiors, 142, 113-135.

Rosenbaum, G., et al. (2006), The dynamic strength profile of the lithosphere: Bridging the gap between continental, passive margin and oceanic detachments, Journal of Geophysical Research, in prep.

Sacks, I., et al. (1978), Slow earthquakes and stress redistribution, Nature, 275, 599-602.

Stein, S., and E. Okal (2005a), Ultra-long period seismic moment of the great December 26, 2004 Sumatra earthquake and implications for the slip process.

Stein, S., and E. A. Okal (2005b), Speed and size of the Sumatra earthquake, Nature, 434, 581.

Wijns, C., et al. (2005), Mode of crustal extension determined by rheological layering, Earth and Planetary Science Letters, 236, 120.

Ziegler, H. (1983), An Introducton to thermomechanics, 2nd Edition ed., 358 pp., NorthHolland Publishing Company, Amsterdam. 
APPENDIX Constitutive equations, corotational stress rate 